\title{
When to Sell an Asset Where Its Drift Drops from a High Value to a Smaller One
}

\author{
Pham Van Khanh \\ Institute of Economics and Corporate Group, Hanoi, Vietnam \\ Email:van_khanh1178@yahoo.com
}

Received 28 June 2015; accepted 8 November 2015; published 11 November 2015

Copyright (C) 2015 by author and Scientific Research Publishing Inc.

This work is licensed under the Creative Commons Attribution International License (CC BY). http://creativecommons.org/licenses/by/4.0/

c) (i) Open Access

\begin{abstract}
To solve the selling problem which is resembled to the buying problem in [1], in this paper we solve the problem of determining the optimal time to sell a property in a location the drift of the asset drops from a high value to a smaller one at some random change-point. This change-point is not directly observable for the investor, but it is partially observable in the sense that it coincides with one of the jump times of some exogenous Poisson process representing external shocks, and these jump times are assumed to be observable. The asset price is modeled as a geometric Brownian motion with a drift that initially exceeds the discount rate, but with the opposite relation after an unobservable and exponentially distributed time and thus, we model the drift as a two-state Markov chain. Using filtering and martingale techniques, stochastic analysis transform measurement, we reduce the problem to a one-dimensional optimal stopping problem. We also establish the optimal boundary at which the investor should liquidate the asset when the price process hit the boundary at first time.
\end{abstract}

\section{Keywords}

Optimal Stopping Time, Posterior Probability, Threshold, Markov Chain, Jump Times, Martingale, Brownian Motion

\section{Introduction}

In this paper we consider the following problem: How to find the optimal stopping time to sell a stock (or an asset) when the expected return of a stock is assumed to be a constant larger than the discount rate up until some random, and unobservable, time $\tau$, at which it drops to a constant smaller than the discount rate.

An investor wants to hold the position as long as the inertia is present by taking advantage of the drift which is exceeding the discounted rate (or interest rate). On the other hand, when the inertia disappears the investor 
would like to exit the position by selling the asset.

The under study problem in this paper was also addressed in [1] where the buying problem with the same assumption was solved. The results of [1] showed that the optimal buying time was the first passage time over some unknown level for the a posteriori probability process $\pi_{t}$ defined below and by simulating it was found that the optimal time to buy an asset was the time which the asset price process had just passed the trough.

The author of [2] studied a problem of finding an optimal stopping strategy to liquidate an asset with unknown drift; more exactly he wanted to find the best time to sell a stock when its drift was a discrete random variable which took the given values. The first time the posterior mean of the drift passes below a non-decreasing boundary that is the unique solution of a particular integral equation is shown to be optimal.

Some classical optimal stopping time problem has been considered in [3]. These are applied in mathematical finance but these are basic problem, and it is difficult to apply in real world.

For related studies of stock selling problems, see [4] [5] and for studies of basic optimal stopping problems see [3]. The method we use to study in this paper is the martingale theory, the transformation theory of measuring and the optimal stopping time is referenced in the literature [3] [6] [7].

In this paper, the asset price is modeled as a linear Brownian motion with a drift that drops from one constant to a smaller constant at some unobservable time. This drift is modeled as a Markov chain with two states which are denoted by 0 and 1 where 0 is denoted for price decrease and 1 is denoted for price increase.

We define the asset price model in Section 2, and the optimal selling problem is set up. In Section 3, we study the simulation to examine our studies and finally, Section 4 is conclusion.

\section{The Model}

We take as given a complete probability space $(\Omega, F, P)$. On this probability space, let the change-point $\tau$ be a random variable with distribution

$$
P(\tau=0)=\pi, P(\tau>t \mid t>0)=\mathrm{e}^{-\lambda t}
$$

where $\lambda$ is the intensity of the transition from state 1 to state 0 and assume that $\lambda$ is positive and that belongs to $[0 ; 1)$. Denote the drift of the price process $a_{t}, t \geq 0$, can be modeled as a Markov chain with two states $a_{l}$ denoted by state 0 and $a_{h}$ denoted by state 1 such that $P\left(a=a_{h}\right)=\pi_{0} ; P\left(a=a_{l}\right)=1-\pi_{0}$ at time $0, a_{l}<r<a_{h}$ where $r$ is discounted rate which is a given constant and process $a_{t}, t \geq 0$ can only transit from state 1 to state 0 with transition density matrix as follows $Q=\left[\begin{array}{cc}0 & 0 \\ \lambda & -\lambda\end{array}\right]$. Next, let $W$ be a Brownian motion which is independent of $\tau$. The asset price process $X$ is modeled by a geometric Brownian motion with a drift that drops from $a_{h}$ to $a_{l}$ at time $\tau$. More precisely,

$$
\mathrm{d} X_{t}=a_{t} X_{t} \mathrm{~d} t+\sigma X_{t} \mathrm{~d} W_{t}
$$

and $X_{0}=x>0$, where $a_{t}=a_{h}-\left(a_{h}-a_{l}\right) I(t \geq \tau)$ i.e. $a_{t}=a_{h}$ if $t<\tau$ and $a_{t}=a_{l}$ if $t \geq \tau$ the volatility $\sigma>0$ is a constant.

At the time of $t>0$ we define the a posteriori probability process $\pi_{t}$ by

$$
\pi_{t}=P\left\{a=a_{l} \mid \mathcal{F}_{t}^{X}\right\}
$$

where $\left\{\mathcal{F}_{t}^{X}\right\}_{0 \leq t \leq T}$ is a filter generated by $X$ and $\tau$. The process $\pi_{t}$ indicate the probability of event that the price process decreases. We consider the optimal stopping problem:

Find $\mathcal{F}^{X}$-stopping time $\tau, 0 \leq \tau \leq T$ such that:

$$
V=\sup _{0 \leq \tau \leq T} E\left[\mathrm{e}^{-r \tau} X_{\tau}\right] .
$$

Similar the buying problem, posterior probability process $\pi_{t}$ satisfying the following stochastic differential (see theorem 9.1, [6]):

$$
\mathrm{d} \pi_{t}=\lambda\left(1-\pi_{t}\right) \mathrm{d} t-\pi_{t} \frac{a_{h} X_{t}-\left[a_{h} \pi_{t}+a_{l}\left(1-\pi_{t}\right)\right] X_{t}}{\sigma X_{t}} \mathrm{~d} \bar{W}_{t}
$$


or

$$
\mathrm{d} \pi_{t}=\lambda\left(1-\pi_{t}\right) \mathrm{d} t-\pi_{t}\left(1-\pi_{t}\right)\left(\frac{a_{h}-a_{l}}{\sigma}\right) \mathrm{d} \bar{W}_{t}
$$

where $d \bar{W}_{t}$ is a $P$-Brownian motion with respect to $\mathcal{F}_{t}^{X}$ given by

$$
\begin{aligned}
\mathrm{d} \bar{W}_{t} & =\frac{\mathrm{d} X_{t}-\left[\left(1-\pi_{t}\right) a_{l}+\pi_{t} a_{h}\right] X_{t} \mathrm{~d} t}{\sigma X_{t}}=\frac{\left\{a_{t}-\left[\left(1-\pi_{t}\right) a_{l}+\pi_{t} a_{h}\right]\right\} X_{t} \mathrm{~d} t+\sigma X_{t} \mathrm{~d} W_{t}}{\sigma X_{t}} \\
& =\frac{\left\{a_{t}-\left[\left(1-\pi_{t}\right) a_{l}+\pi_{t} a_{h}\right]\right\}}{\sigma} \mathrm{d} t+\mathrm{d} W_{t} .
\end{aligned}
$$

Moreover, in terms of $\bar{W}_{t}$ we have

$$
\frac{\mathrm{d} X_{t}}{X_{t}}=E\left[a_{t} \mid \mathcal{F}_{t}^{X}\right] \mathrm{d} t+\sigma \mathrm{d} \bar{W}_{t}=\left[\left(1-\pi_{t}\right) a_{l}+\pi_{t} a_{h}\right] \mathrm{d} t+\sigma \mathrm{d} \bar{W}_{t} .
$$

Processes $X_{t}$ and $\pi_{t}$ satisfy the following system equations:

$$
\left\{\begin{array}{l}
\frac{\mathrm{d} X_{t}}{X_{t}}=\left(a_{l}+\pi_{t}\left(a_{h}-a_{l}\right)\right) \mathrm{d} t+\sigma \mathrm{d} \bar{W}_{t} \\
\mathrm{~d} \pi_{t}=\lambda\left(1-\pi_{t}\right) \mathrm{d} t+\pi_{t}\left(1-\pi_{t}\right)\left(\frac{a_{h}-a_{l}}{\sigma}\right) \mathrm{d} \bar{W}_{t} .
\end{array}\right.
$$

Put $\Phi_{t}=\frac{\pi_{t}}{1-\pi_{t}} ; \omega=\frac{a_{h}-a_{l}}{\sigma}$ and Ito's formula gives that:

$$
\mathrm{d} \Phi_{t}=\left(\frac{\lambda_{2}}{1-\pi_{t}}+\omega^{2} \pi_{t} \Phi_{t}\right) \mathrm{d} t-\omega \Phi_{t} \mathrm{~d} \bar{W}_{t}
$$

We define new process $\left\{\tilde{W}_{t}\right\}$ as follow:

$$
\mathrm{d} \tilde{W}_{t}=-\left(\omega \pi_{t}+\sigma\right) \mathrm{d} t+\mathrm{d} \bar{W}_{t}
$$

and a new measure $P^{*}$ satisfying:

$$
\begin{aligned}
\frac{\mathrm{d} P^{*}}{\mathrm{~d} P} & =\exp \left\{\frac{1}{2} \int_{0}^{T}\left(\sigma+\omega \pi_{t}\right)^{2} \mathrm{~d} t-\int_{0}^{T}\left(\sigma+\omega \pi_{t}\right) \mathrm{d} \bar{W}_{t}\right\} \\
& =\exp \left\{-\frac{1}{2} \int_{0}^{T}\left(\sigma+\omega \pi_{t}\right)^{2} \mathrm{~d} t-\int_{0}^{T}\left(\sigma+\omega \pi_{t}\right) \mathrm{d} \tilde{W}_{t}\right\} .
\end{aligned}
$$

By Girsanov theorem, $\tilde{W}_{t}$ is a $P^{*}$-Brownian motion. Furthermore,

$$
\mathrm{d} \Phi_{t}=\left(\lambda+(\lambda-\sigma \omega) \Phi_{t}\right) \mathrm{d} t-\omega \Phi_{t} \mathrm{~d} \tilde{W}_{t} \Rightarrow \Phi_{t}=Z_{t}\left[\Phi_{0}+\lambda \int_{0}^{t} Z_{s}^{-1} \mathrm{~d} s\right]
$$

where

$$
Z_{t}=\exp \left\{\left(\lambda_{2}+\sigma \omega-\frac{\omega^{2}}{2}\right) t-\omega \tilde{W}_{t}\right\} .
$$

The price process $X_{t}$ satisfying the following stochastic differential

$$
\frac{\mathrm{d} X_{t}}{X_{t}}=\left[\left(1-\pi_{t}\right) a_{h}+\pi_{t} a_{l}\right] \mathrm{d} t+\sigma\left(\mathrm{d} \tilde{W}_{t}+\left(\omega \pi_{t}+\sigma\right) \mathrm{d} t\right)
$$


or in term of $\tilde{W}_{t}$

$$
\frac{\mathrm{d} X_{t}}{X_{t}}=\left(a_{h}+\sigma^{2}\right) \mathrm{d} t+\sigma \mathrm{d} \tilde{W}_{t} .
$$

The solution of this stochastic equation is

$$
X_{t}=X_{0} \exp \left\{\left(a_{h}+\frac{\sigma^{2}}{2}\right) t+\sigma \tilde{W}_{t}\right\} .
$$

Now we consider the process:

$$
\eta_{t}=\exp \left\{-\frac{1}{2} \int_{0}^{t}\left(\sigma-\omega \pi_{t}\right)^{2} \mathrm{~d} t-\int_{0}^{t}\left(\sigma-\omega \pi_{t}\right) \mathrm{d} \tilde{W}_{t}\right\}
$$

then $\eta_{t}$ is a $\mathcal{F}^{X}$-martingale and $\frac{\mathrm{d} \eta_{t}}{\eta_{t}}=-\left(\omega \pi_{t}+\sigma\right) \mathrm{d} \tilde{W}_{t}$ where $\eta_{0}=1$.

Let $U_{t}$ is a process which defined by $\mathrm{d} U_{t}=-\lambda U_{t} \mathrm{~d} t-\sigma U_{t} \mathrm{~d} \tilde{W}_{t}$, we have $U_{t}=\frac{\mathrm{e}^{\left(a_{h}-\lambda\right) t}}{X_{t}}, U_{0}=\frac{1}{X_{0}}$.

Put $Y_{t}=\frac{\left(1+\Phi_{t}\right) U_{t}}{\left(1+\Phi_{0}\right) U_{0}}, Y_{0}=1$ and according to Itô's formula:

$$
\frac{\mathrm{d} Y_{t}}{Y_{t}}=-\left(\omega \pi_{t}+\sigma\right) \mathrm{d} \tilde{W}_{t}
$$

From this we have $Y_{t}=\eta_{t} \forall t$ (a.s.), thus:

$$
\mathrm{e}^{-r \tau} \eta_{\tau} X_{\tau}=\mathrm{e}^{-r \tau} Y_{\tau} X_{\tau}=\frac{X_{0}}{1+\Phi_{0}} \mathrm{e}^{\left(a_{h}-\lambda-r\right) \tau}\left(1+\Phi_{\tau}\right)
$$

Denote

$$
X_{0}=x, \Phi_{0}=\phi
$$

then

$$
E_{P} \mathrm{e}^{-r \tau} X_{\tau}=E_{P^{*}} \eta_{T} \mathrm{e}^{-r \tau} X_{\tau}=E_{P^{*}} \eta_{\tau} \mathrm{e}^{-r \tau} X_{\tau}=\frac{X}{1+\phi} E_{P^{*}} \mathrm{e}^{\left(a_{h}-\lambda-r\right) \tau}\left(1+\Phi_{\tau}\right) .
$$

To solve the problem (2.1) we solve the following auxiliary problem:

$$
G(\phi)=\sup _{\tau \in \mathfrak{M}} E_{P^{*}} \mathrm{e}^{\left(a_{h}-\lambda-r\right) \tau}\left(1+\Phi_{t}\right) .
$$

Put

$$
Z_{t}=\mathrm{e}^{\left(a_{h}-\lambda-r\right) \tau}\left(1+\Phi_{t}\right) .
$$

The optimal stopping time is the first hitting time of the process $\Phi_{t}$ to the area $[B, \infty)$ with some $B$. Moreover pairs $(B, F)$ satisfying the flowing free boundary problem:

$$
\begin{cases}\mathcal{L} G+\left(a_{h}-\lambda-r\right) G=0 & 0<z<B \\ G(z)=1+z & z \geq B \\ G^{\prime}(B)=1 & \\ G(0+)<\infty & \end{cases}
$$

where $\mathcal{L}$ is infinitesimal generated operator.

Differential equation in (2.3) has the general solution as follows: $G(z)=C_{1} G_{1}(z)+C_{2} G_{2}(z)$ where 


$$
\begin{gathered}
G_{1}(x)=x \mathrm{e}^{\frac{\lambda-\lambda x \ln x+\omega \sigma x \ln x}{\omega^{2} x}} \text { Whittaker } W\left(\frac{\omega^{2}+\omega \sigma-\lambda}{\omega^{2}},-\frac{\sqrt{4 \omega^{2} \lambda-4 \omega^{2}\left(a_{h}+a_{l}-2 r\right)+\omega^{4}+4\left(a_{h}-a_{l}-\lambda\right)^{2}}}{2 \omega^{2}}, \frac{2 \lambda}{\omega^{2} x}\right) \\
G_{2}(x)=x \mathrm{e}^{\frac{\lambda-\lambda x \ln x+\omega \sigma x \ln x}{\omega^{2} x}} \text { Whittaker } M\left(\frac{\omega^{2}+\omega \sigma-\lambda}{\omega^{2}},-\frac{\sqrt{4 \omega^{2} \lambda-4 \omega^{2}\left(a_{h}+a_{l}-2 r\right)+\omega^{4}+4\left(a_{h}-a_{l}-\lambda\right)^{2}}}{2 \omega^{2}}, \frac{2 \lambda}{\omega^{2} x}\right) .
\end{gathered}
$$

Changing variables and using some analytic transformations we obtain:

$$
\alpha=\frac{2 \lambda}{\omega^{2}}>0, \beta=\alpha-\frac{2 \sigma}{\omega}>0, \gamma=\sqrt{(\beta-1)^{2}+\frac{8\left(\lambda+r-a_{h}\right)}{\omega^{2}}}>|\beta-1|
$$

then

$$
G_{1}(z)=\int_{0}^{\infty} \mathrm{e}^{-\alpha u} u^{(\beta+\gamma-3) / 2}(1+z u)^{(\gamma-\beta+1) / 2} \mathrm{~d} u \text { and } G_{2}(z)=\int_{0}^{\frac{1}{2}} \mathrm{e}^{\alpha u} u^{(\beta+\gamma-3) / 2}(1-z u)^{(\gamma-\beta+1) / 2} \mathrm{~d} u .
$$

We also have

$$
G_{2}(z)>(1-\sqrt{z})^{(\gamma-\beta+1) / 2} \int_{0}^{\frac{1}{\sqrt{z}}} u^{(\beta+\gamma-3) / 2} \mathrm{~d} u=\frac{2}{\beta+\gamma-1}(1-\sqrt{z})^{(\gamma-\beta+1) / 2} z^{-\frac{(\beta+\gamma-1)}{4}}>\varepsilon z^{-\frac{(\beta+\gamma-1)}{4}} \rightarrow+\infty
$$

as $z \rightarrow 0+$ since $0<z<1$ and $-\frac{\beta+\gamma-1}{4}<0$.

We have

$$
G_{1}^{\prime}(z)=\frac{\gamma-\beta+1}{2} \int_{0}^{\infty} \mathrm{e}^{-\alpha t} t^{(\beta+\gamma-1) / 2}(1+z t)^{(\gamma-\beta-1) / 2} \mathrm{~d} t>0
$$

since $r+\lambda>a_{h}$ therefore $\gamma=\sqrt{(\beta-1)^{2}+\frac{8\left(\lambda+r-a_{h}\right)}{\omega^{2}}}>\beta-1$ and $G_{1}(z)$ is an increasing function.

Moreover

$$
G_{1}^{\prime \prime}(z)=\frac{\gamma-\beta+1}{2} \frac{\gamma-\beta-1}{2} \int_{0}^{\infty} \mathrm{e}^{-\alpha t} t^{(\beta+\gamma+1) / 2}(1+z t)^{(\gamma-\beta-3) / 2} \mathrm{~d} t<0
$$

since

$$
\begin{aligned}
\gamma & =\sqrt{(\beta-1)^{2}+\frac{8\left(\lambda+r-a_{h}\right)}{\omega^{2}}}>\sqrt{(\beta-1)^{2}+\frac{8\left(\lambda+a_{l}-a_{h}\right)}{\omega^{2}}} \\
& =\sqrt{(\beta-1)^{2}+\frac{4 \times 2(\lambda-\sigma \omega)}{\omega^{2}}}=\sqrt{(\beta-1)^{2}+4 \beta}=\beta+1 .
\end{aligned}
$$

These mean that the function $G_{1}(z)$ is increasing and convex on $(0, \infty)$.

Figure 1 shows the graph of function $G_{1}(z)$, we can check the increase and convex properties of it. The graph of $G_{2}(z)$ is shown in Figure 2, we can see that it tends to infinite when $z$ as $0+$.

But $G(0+)<\infty$ therefore $C_{2}=0$ and $G(z)=C_{1} \int_{0}^{\infty} \mathrm{e}^{-\alpha u} u^{(\beta+\gamma-3) / 2}(1+z u)^{(\gamma-\beta+1) / 2} \mathrm{~d} u$.

According to (2.3) we have

$$
\left\{\begin{array}{l}
C_{1} \int_{0}^{\infty} \mathrm{e}^{-\alpha u} u^{(\beta+\gamma-3) / 2}(1+B u)^{(\gamma-\beta+1) / 2} \mathrm{~d} u=1+B \\
C_{1} \frac{\gamma-\beta+1}{2} \int_{0}^{\infty} \mathrm{e}^{-\alpha t} u^{(\beta+\gamma-1) / 2}(1+B u)^{(\gamma-\beta-1) / 2} \mathrm{~d} u=1
\end{array}\right.
$$




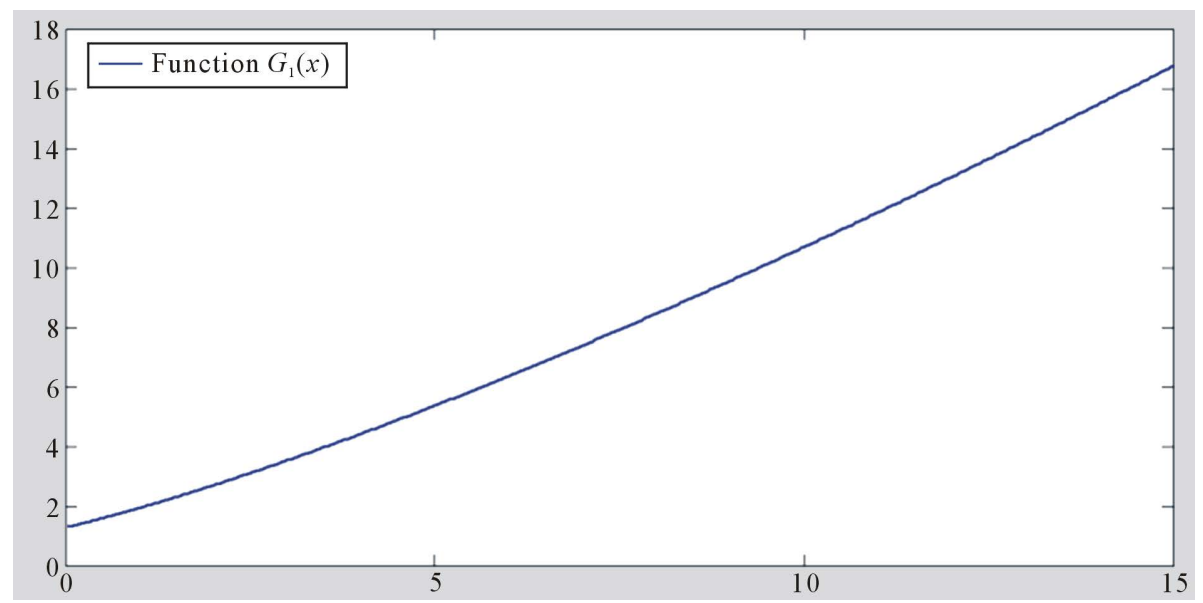

Figure 1. Graph of the function $G_{1}(x)$.

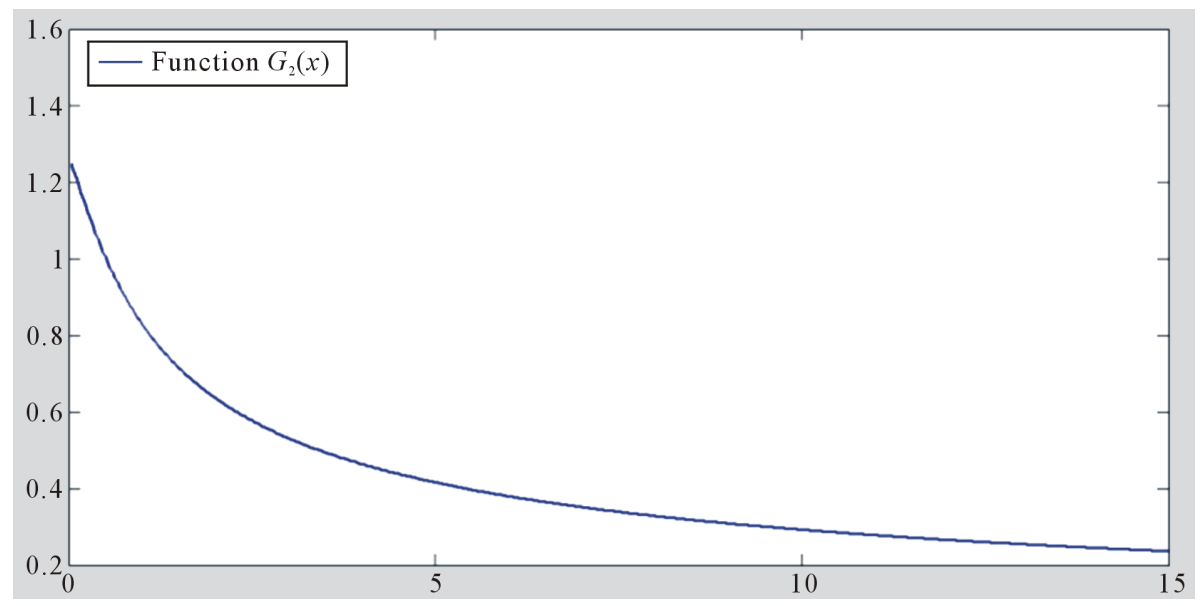

Figure 2. Graph of the function $G_{2}(x)$.

So $B$ is the solution of the following equation:

$$
2 \int_{0}^{\infty} \mathrm{e}^{-\alpha u} u^{(\beta+\gamma-3) / 2}(1+B u)^{(\gamma-\beta+1) / 2} \mathrm{~d} u=(1+B)(\gamma-\beta+1) \int_{0}^{\infty} \mathrm{e}^{-\alpha u} u^{(\beta+\gamma-1) / 2}(1+B u)^{(\gamma-\beta-1) / 2} \mathrm{~d} u .
$$

\section{Lemma 2.1. The free boundary Equation (2.4) has unique positive solution B.}

Proof: The Equation (2.4) is equivalent to

$$
2 \int_{0}^{\infty} \mathrm{e}^{-\alpha u} u^{(\beta+\gamma-3) / 2}(1+x u)^{(\gamma-\beta+1) / 2} \mathrm{~d} u-(1+x)(\gamma-\beta+1) \int_{0}^{\infty} \mathrm{e}^{-\alpha u} u^{(\beta+\gamma-1) / 2}(1+x u)^{(\gamma-\beta-1) / 2} \mathrm{~d} u=0 .
$$

Denote:

$$
h(x)=2 \int_{0}^{\infty} \mathrm{e}^{-\alpha u} u^{(\beta+\gamma-3) / 2}(1+x u)^{(\gamma-\beta+1) / 2} \mathrm{~d} u-(1+x)(\gamma-\beta+1) \int_{0}^{\infty} \mathrm{e}^{-\alpha u} u^{(\beta+\gamma-1) / 2}(1+x u)^{(\gamma-\beta-1) / 2} \mathrm{~d} u .
$$

The graph of $h(x)$ is shown in Figure 3. We shall prove that the function $h(x)$ satisfying $\lim _{x \rightarrow 0+} h(x)>0$, $\lim _{x \rightarrow+\infty} h(x)=-\infty$ and $h(x)$ is decreasing and therefore the equation $h(x)=0$ has unique solution on $(0,+\infty)$. We have 


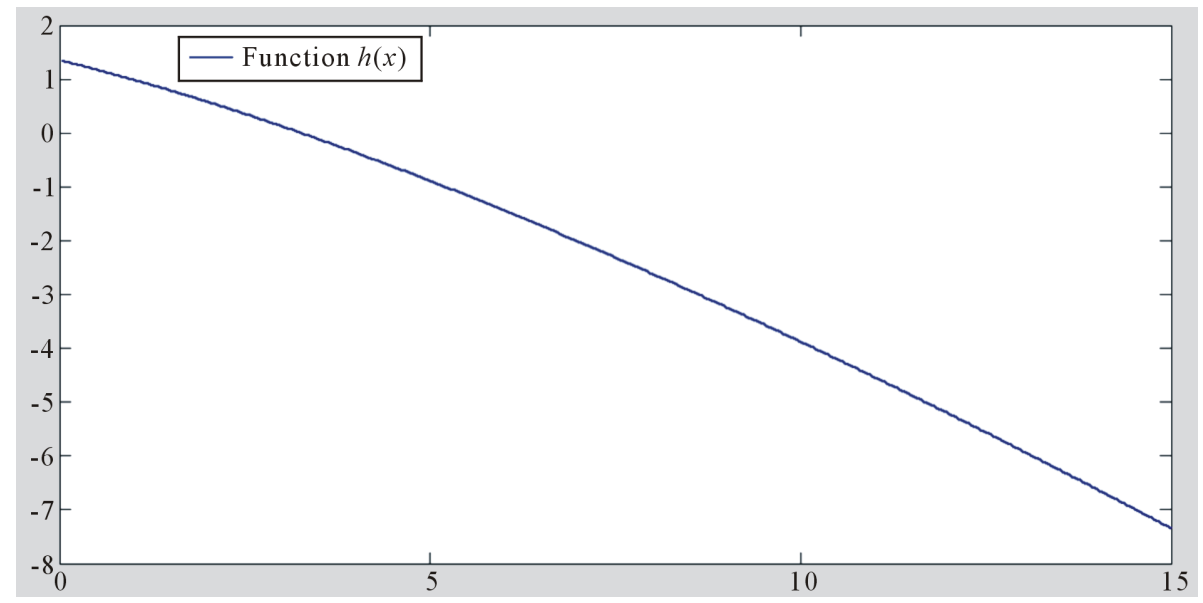

Figure 3. Graph of the function $h(x)$.

$$
\begin{aligned}
h(0+) & =2 \int_{0}^{\infty} \mathrm{e}^{-\alpha u} u^{(\beta+\gamma-3) / 2} \mathrm{~d} u-(\gamma-\beta+1) \int_{0}^{\infty} \mathrm{e}^{-\alpha u} u^{(\beta+\gamma-1) / 2} \mathrm{~d} u \\
& =2 \int_{0}^{\infty} \mathrm{e}^{-\alpha u} u^{(\beta+\gamma-3) / 2} \mathrm{~d} u-(\gamma-\beta+1) \int_{0}^{\infty} \mathrm{e}^{-\alpha u} u^{(\beta+\gamma-1) / 2} \mathrm{~d} u \\
& =\frac{4}{\beta+\gamma-1} \int_{0}^{\infty} \mathrm{e}^{-\alpha u} \mathrm{~d} u^{(\beta+\gamma-3) / 2}-(\gamma-\beta+1) \int_{0}^{\infty} \mathrm{e}^{-\alpha u} u^{(\beta+\gamma-1) / 2} \mathrm{~d} u \\
& =\frac{4}{\beta+\gamma-1}\left[\left.\mathrm{e}^{-\alpha u} u^{(\beta+\gamma-1) / 2}\right|_{0} ^{\infty}+\alpha \int_{0}^{\infty} \mathrm{e}^{-\alpha u} u^{(\beta+\gamma-1) / 2} \mathrm{~d} u\right]-(\gamma-\beta+1) \int_{0}^{\infty} \mathrm{e}^{-\alpha u} u^{(\beta+\gamma-1) / 2} \mathrm{~d} u \\
& =\left[\frac{4 \alpha}{\beta+\gamma-1}-(\gamma-\beta+1)\right] \int_{0}^{\infty} \mathrm{e}^{-\alpha u} u^{(\beta+\gamma-1) / 2} \mathrm{~d} u .
\end{aligned}
$$

It follows that

$$
\begin{aligned}
h(0+) & =\left[\frac{4 \alpha}{\beta-1+\gamma}+(\beta-1-\gamma)\right] \int_{0}^{\infty} \mathrm{e}^{-\alpha u} u^{(\beta+\gamma-1) / 2} \mathrm{~d} u=\left[\frac{4 \alpha+(\beta-1)^{2}-\gamma^{2}}{\beta-1+\gamma}\right] \int_{0}^{\infty} \mathrm{e}^{-\alpha u} u^{(\beta+\gamma-1) / 2} \mathrm{~d} u \\
& =\left[\frac{\frac{8 \lambda}{\omega^{2}}-\frac{8\left(\lambda+r-a_{h}\right)}{\omega^{2}}}{\beta-1+\gamma}\right] \int_{0}^{\infty} \mathrm{e}^{-\alpha u} u^{(\beta+\gamma-1) / 2} \mathrm{~d} u=\frac{8\left(a_{h}-r\right)}{\omega^{2}(\beta-1+\gamma)} \int_{0}^{\infty} \mathrm{e}^{-\alpha u} u^{(\beta+\gamma-1) / 2} \mathrm{~d} u>0 \\
h^{\prime}(x)= & (\gamma-\beta+1) \int_{0}^{\infty} \mathrm{e}^{-\alpha u} u^{(\beta+\gamma-1) / 2}(1+x u)^{(\gamma-\beta-1) / 2} \mathrm{~d} u-(\gamma-\beta+1) \int_{0}^{\infty} \mathrm{e}^{-\alpha u} u^{(\beta+\gamma+1) / 2}(1+x u)^{(\gamma-\beta-1) / 2} \mathrm{~d} u \\
& -(1+x) \frac{\gamma-\beta-1}{2}(\gamma-\beta+1) \int_{0}^{\infty} \mathrm{e}^{-\alpha u} u^{(\beta+\gamma+1) / 2}(1+x u)^{(\gamma-\beta-3) / 2} \mathrm{~d} u \\
= & -(1+x) \frac{\gamma-\beta-1}{2}(\gamma-\beta+1) \int_{0}^{\infty} \mathrm{e}^{-\alpha u} u^{(\beta+\gamma+1) / 2}(1+x u)^{(\gamma-\beta-3) / 2} \mathrm{~d} u .
\end{aligned}
$$

Because

$$
\alpha=\frac{2 \lambda}{\omega^{2}}>0, \beta=\alpha-\frac{2 \sigma}{\omega}=\frac{2\left(\lambda+a_{l}-a_{h}\right)}{\omega^{2}}>0, \gamma=\sqrt{(\beta-1)^{2}+\frac{8\left(\lambda+r-a_{h}\right)}{\omega^{2}}}>|\beta-1|
$$

and 


$$
\gamma=\sqrt{(\beta-1)^{2}+\frac{8\left(\lambda+r-a_{h}\right)}{\omega^{2}}}>\sqrt{(\beta-1)^{2}+\frac{8\left(\lambda+a_{l}-a_{h}\right)}{\omega^{2}}}=\sqrt{(\beta-1)^{2}+4 \beta}=\beta+1
$$

we obtain

$$
h^{\prime}(x)=-(1+x) \frac{\gamma-\beta-1}{2}(\gamma-\beta+1) \int_{0}^{\infty} \mathrm{e}^{-\alpha u} u^{(\beta+\gamma+1) / 2}(1+x u)^{(\gamma-\beta-3) / 2} \mathrm{~d} u<0 \quad \forall x>0 .
$$

We will prove that $\lim _{x \rightarrow+\infty} h(x)=-\infty$. Indeed, with the large enough $x$ we have $1+x u \sim x u$ so

$$
\begin{aligned}
h(x) & =2 \int_{0}^{\infty} \mathrm{e}^{-\alpha u} u^{(\beta+\gamma-3) / 2}(1+x u)^{(\gamma-\beta+1) / 2} \mathrm{~d} u-(1+x)(\gamma-\beta+1) \int_{0}^{\infty} \mathrm{e}^{-\alpha u} u^{(\beta+\gamma-1) / 2}(1+x u)^{(\gamma-\beta-1) / 2} \mathrm{~d} u \\
& \sim 2 x^{(\gamma-\beta+1) / 2} \int_{0}^{\infty} \mathrm{e}^{-\alpha u} u^{(\beta+\gamma-3) / 2} u^{(\gamma-\beta+1) / 2} \mathrm{~d} u-(1+x)(\gamma-\beta+1) x^{(\gamma-\beta-1) / 2} \int_{0}^{\infty} \mathrm{e}^{-\alpha u} u^{(\beta+\gamma-1) / 2} u^{(\gamma-\beta-1) / 2} \mathrm{~d} u \\
& =2 x^{(\gamma-\beta+1) / 2} \int_{0}^{\infty} \mathrm{e}^{-\alpha u} u^{(\beta+\gamma-3) / 2} u^{(\gamma-\beta+1) / 2} \mathrm{~d} u-(\gamma-\beta+1) x^{(\gamma-\beta+1) / 2} \int_{0}^{\infty} \mathrm{e}^{-\alpha u} u^{\gamma-1} \mathrm{~d} u \\
& =(1+\beta-\gamma) x^{(\gamma-\beta+1) / 2} \int_{0}^{\infty} \mathrm{e}^{-\alpha u} u^{\gamma-1} \mathrm{~d} u \rightarrow-\infty
\end{aligned}
$$

since $1+\beta-\gamma<0, \gamma-\beta+1>0, \int_{0}^{\infty} \mathrm{e}^{-\alpha u} u^{\gamma-1} \mathrm{~d} u=$ const $>0$.

Consequently, $\lim _{x \rightarrow 0+} h(x)>0, \lim _{x \rightarrow+\infty} h(x)=-\infty$ and $h(x)$ is decreasing so the equation $h(x)=0$ has unique solution on $(0,+\infty)$. The theorem is proved. $\square$

Theorem 2.2. Stopping time $\tau_{B}=\inf \left\{t \geq 0: \Phi_{t} \geq B\right\}$ is the optimal stopping time for (2.1).

Proof: Let

$$
L(x)= \begin{cases}\frac{1+B}{G_{1}(B)} G_{1}(x) & x<B \\ 1+x & x \geq B\end{cases}
$$

and we will prove that $L(x)>1+x \quad \forall x<B$, indeed

$$
\frac{1+B}{G_{1}(B)} G_{1}(x)>1+x \Leftrightarrow \frac{1+B}{G_{1}(B)}>\frac{1+x}{G_{1}(x)} .
$$

Now, we examine the function $g(x)=\frac{1+x}{G_{1}(x)}$.

Take the derivative we obtain

$$
\begin{aligned}
g^{\prime}(x) & =\frac{1}{F_{1}^{2}(x)}\left(\int_{0}^{\infty} \mathrm{e}^{-\alpha u} u^{(\beta+\gamma-3) / 2}(1+x u)^{(\gamma-\beta+1) / 2} \mathrm{~d} u-(1+x) \frac{\gamma-\beta+1}{2} \int_{0}^{\infty} \mathrm{e}^{-\alpha u} u^{(\beta+\gamma-1) / 2}(1+x u)^{(\gamma-\beta-1) / 2} \mathrm{~d} u\right) \\
& =\frac{h(x)}{F_{1}^{2}(x)}>\frac{h(B)}{F_{1}^{2}(B)}=0 \Rightarrow g(B)>g(x) \Rightarrow \frac{1+B}{G_{1}(B)}>\frac{1+x}{G_{1}(x)} .
\end{aligned}
$$

This follows

$$
L(x) \geq 1+x \quad \forall x .
$$

Using the Dynkin's formula to the process $Y_{t}=\mathrm{e}^{\left(a_{h}-\lambda-r\right) t} L\left(\Phi_{t}\right)$ we have:

$$
\mathrm{d} Y_{t}=\mathrm{e}^{\left(a_{h}-\lambda-r\right) t}\left(a_{h}-r+\left(a_{l}-r\right) \Phi_{t}\right) 1_{\left\{\Phi_{t}>B\right\}} \mathrm{d} t-\mathrm{e}^{\left(a_{h}-\lambda-r\right) t} \omega \Phi_{t} L^{\prime}\left(\Phi_{t}\right) \mathrm{d} \tilde{W}_{t} .
$$


Because $B$ satisfying $B \geq \frac{a_{h}-r}{r-a_{l}}:=d$ so the drift of $Y$ is positive and therefore $Y$ is super martingale and $Y_{t \wedge \tau_{B}}$ is martingale. By optional theorem we have:

$$
\mathbb{E}_{\phi} \mathrm{e}^{\left(a_{h}-\lambda-r\right) \tau}\left(1+\Phi_{\tau}\right) \leq \mathbb{E}_{\phi} Y_{\tau} \leq \mathbb{E}_{\phi} Y_{0}=L(\phi) \Rightarrow G(\phi) \leq L(\phi) .
$$

By $\tau=\tau_{B}$ we have $\mathbb{E}_{\phi} Y_{\tau_{B}}=\mathbb{E}_{\phi} Y_{0}=L(\phi)$ moreover

$$
G(\phi)=\sup _{\tau} \mathbb{E}_{y}^{Q} Y_{\tau} \geq \mathbb{E}_{y}^{Q} Y_{\tau_{B}}=L(\phi) \text {. So } G(\phi)=L(\phi) \text {. }
$$

We will show that $B$ satisfy the condition: $B \geq \frac{a_{h}-r}{r-a_{l}}:=d$. Indeed, by general optimal stopping theory all points satisfy the form

$$
\left(\mathcal{L}+a_{h}-\lambda-r\right)(1+\phi)=a_{h}-r+\left(a_{l}-r\right) \phi
$$

with positive value will be in continuation area

$$
C=\{\phi: G(\phi)>1+\phi\} .
$$

The optimal stopping time is the first hitting time of $\left\{\Phi_{t}\right\}_{t \geq 0}$ to the area:

$$
D=\{\phi: G(\phi)=1+\phi\} \text { or } \tau_{B}=\inf \left\{t \geq 0: \Phi_{t} \geq B\right\} .
$$

Thus function $G$ satisfy the following condition:

$$
G(\phi)=\sup _{\tau: \Phi_{t} \geq d} \mathbb{E}_{\phi} \mathrm{e}^{\left(a_{h}-\lambda-r\right)}\left(1+\Phi_{\tau}\right) .
$$

We define the function:

$$
G^{d}(\phi)= \begin{cases}\frac{1+d}{G_{1}(\phi)} G_{1}(\phi) & \phi<d \\ 1+\phi & \phi \geq d\end{cases}
$$

Now, we assume $B<d$, because $h(x)$ is a decreasing function so $h(d)<0$. Then the left derivative of $\left(G^{d}(d-)\right)^{\prime}>\left(G^{d}(d+)\right)^{\prime}$. Specially, $G^{d}(\phi)<1+\phi$ with some $\phi<d$. This follows $\mathrm{e}^{\left(a_{h}-\lambda_{2}-r\right) t} G^{d}\left(\Phi_{t}\right)$ is super martingale and $\mathrm{e}^{\left(a_{h}-\lambda-r\right) t \wedge \tau_{d}} G^{d}\left(\Phi_{t \wedge \tau_{d}}\right)$ martingale. For the stopping time $\tau$ satisfying $\Phi_{\tau} \geq d$ we have:

$$
\mathbb{E}_{\phi} \mathrm{e}^{\left(a_{h}-\lambda-r\right)}\left(1+\Phi_{\tau}\right)=\mathbb{E}_{\phi} e^{\left(a_{h}-\lambda-r\right)} G^{d}\left(\Phi_{\tau}\right) \leq G^{d}(\phi) \Rightarrow G(\phi) \leq G^{d}(\phi) .
$$

This contradicts to the existence of $\phi$ such that $G^{d}(\phi)<1+\phi$ when $G(\phi) \geq 1+\phi \quad \forall \phi$. Finally, we achieve $B \geq d$.

The optimal stopping time $\tau_{B}$ is the first hitting time of $\left\{\Phi_{t}\right\}_{t \geq 0}$ to the area

$$
D=\{\phi: G(\phi)=1+\phi\} \text {. }
$$

But $G(\phi) \geq 1+\phi \quad \forall \phi$ therefore

$$
\tau_{B}=\inf \left\{t \geq 0: \Phi_{t} \geq B\right\},
$$

by this, we have finished the provement.

\section{Simulation Study}

To make visual for the above theory we simulate the asset price process, the posterior probability process $\pi_{t}$ process $\Phi(t)=\frac{\pi_{t}}{1-\pi_{t}}$ (notice that $\Phi(t)$ is an increasing function of $\pi_{t}$ ) and the selling threshold $\boldsymbol{B}$. Some 
parameters is used in our simulating are $a_{l}=-0.05 ; r=0.06 ; a_{h}=0.2 ; \sigma=0.04 ; \lambda=0.5$ and the time interval is $[0,1]$.

As can be seen in the figures from 4 to 8 if the price is increasing then the $\Phi(t)$ and $\pi_{t}$ are decreasing and conversely.

Figure 4 shows the price process has increased since the time 0.2 so the $\Phi(t)$ decreased from the respective time and it can not hit the red line denoted the threshold, therefore the optimal selling time in this case is 1 .

Figure 5 simulate a price process which is fluctuated from time 0 to 0.14 and decrease dramatically at the time 0.14 so the process $\Phi(t)$ increase sharply from this and crossover the threshold, it follows that the optimal time to liquidate the asset is about 0.17 . At this time the price of the asset is lower than the origin but if we hold it we will sell it at a much more loss in the future.

Another simulation is shown in Figure 6. Clearly, whenever the price process is increasing, the $\Phi(t)$ and the posterior probability process are decreasing and the liquidated time is 0.77 . At this time the price is not the highest but it is significantly higher than the original value which is 1.5 .

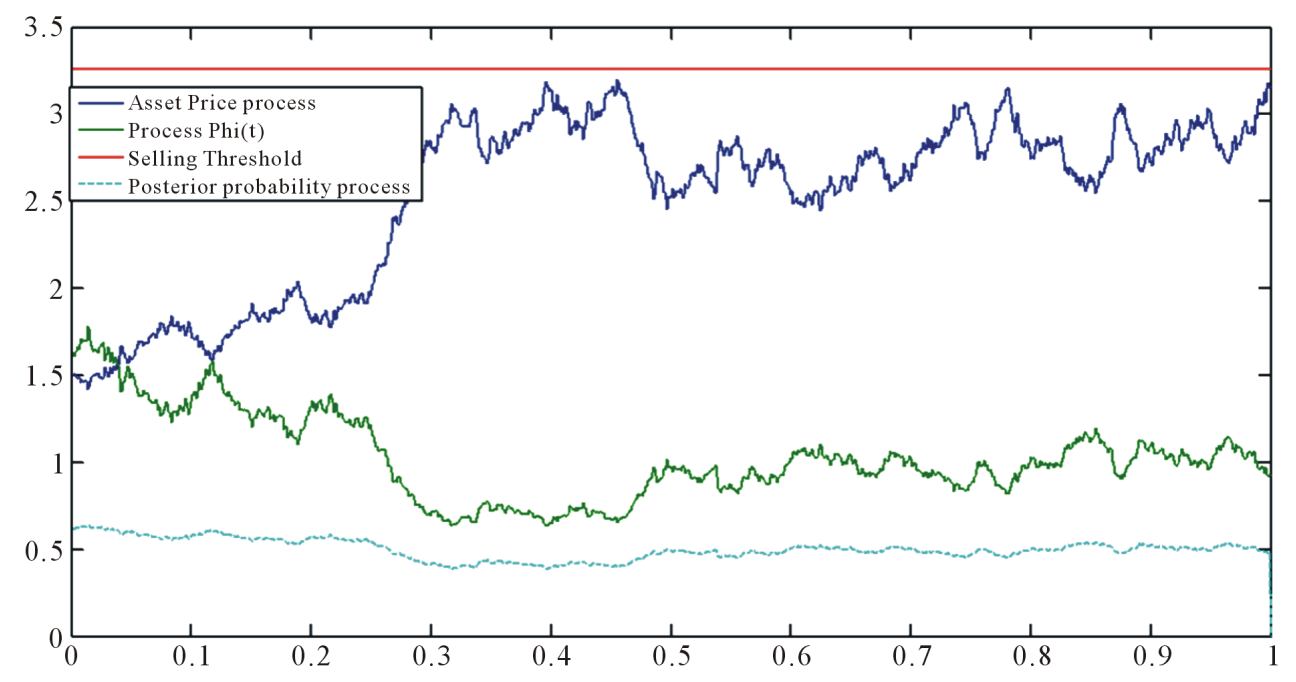

Figure 4. A simulation of asset price process, the posterior probability process, process $\Phi(t)$, the threshold probability and the optimal stopping time. In this case, the process $\Phi(t)$ always under the threshold probability so the optimal stopping time is the final time 1.

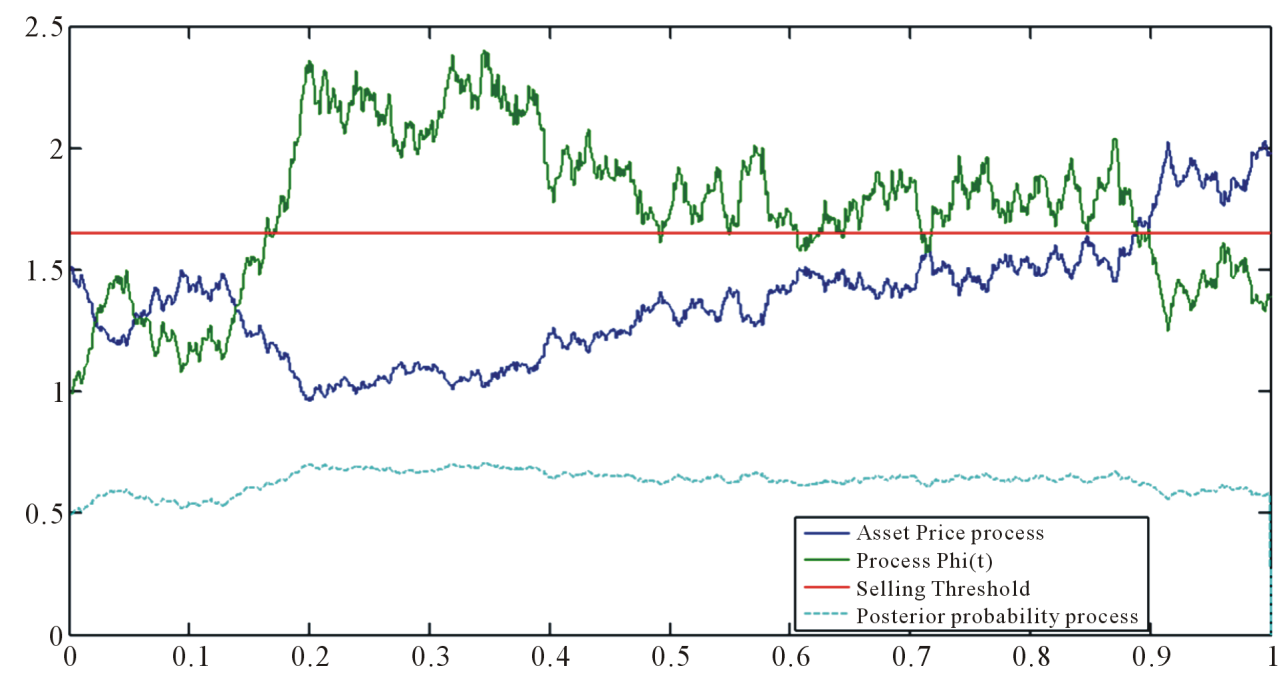

Figure 5. A simulation of asset price process, the posterior probability process, process $\Phi(t)$, the threshold probability and the optimal stopping time. In this case, the first time that the process $\Phi(t)$ over passes the threshold probability at the time 0.17 so the optimal stopping time is 0.17 . 
In Figure 7, we can see the same scenario with the simulation in Figure 6. The time to liquidate in this case is 0.795 , the price is about 1.75 whereas the started price was 1.5 . We can see $S_{t} \mathrm{e}^{-r t}=1.75 * \mathrm{e}^{-0.06 * 0.795}=1.668>1.5=S_{0}$ it means that we benefit by this trade affair.

The same scenario with the simulation in Figure 1, the simulation results in Figure 8 show the price illustrates an uptrend from time 0 to the end that the process $\Phi(t)$ can not pass over the selling threshold B, consequently, the optimal time to sell in this situation is 1.

\section{Conclusion}

This research considers the problem of how to find the optimal time to liquidate an asset when the asset price is modeled by the geometric Brownian motion which has a change point. In particular, the drift of the process drops from a high value to a smaller one and this drift process can be modeled as two-state Markov process. The results of this research indicate that a optimal selling decision is made when the probability of downtrend surpassed some certain threshold. We also simulate the price process with a number of parameters and conduct

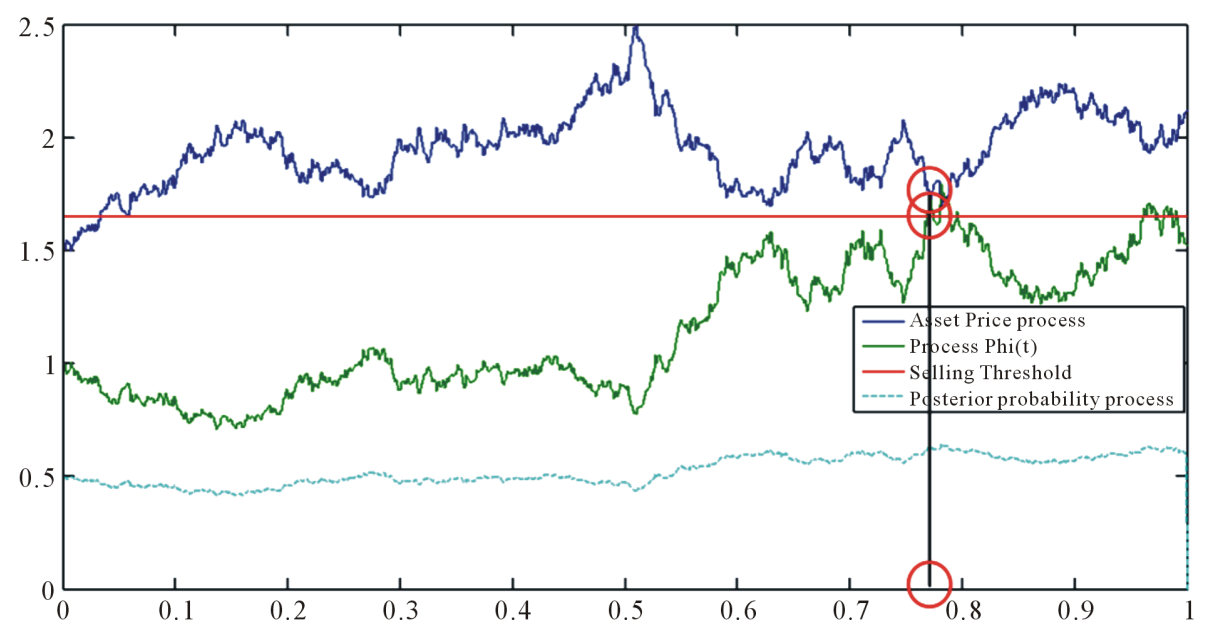

Figure 6. A simulation of asset price process, the posterior probability process, process $\Phi(t)$, the threshold probability and the optimal stopping time. In this case, the first time that the process $\Phi(t)$ over passes the threshold probability at the time 0.77 so the optimal stopping time is 0.77 .

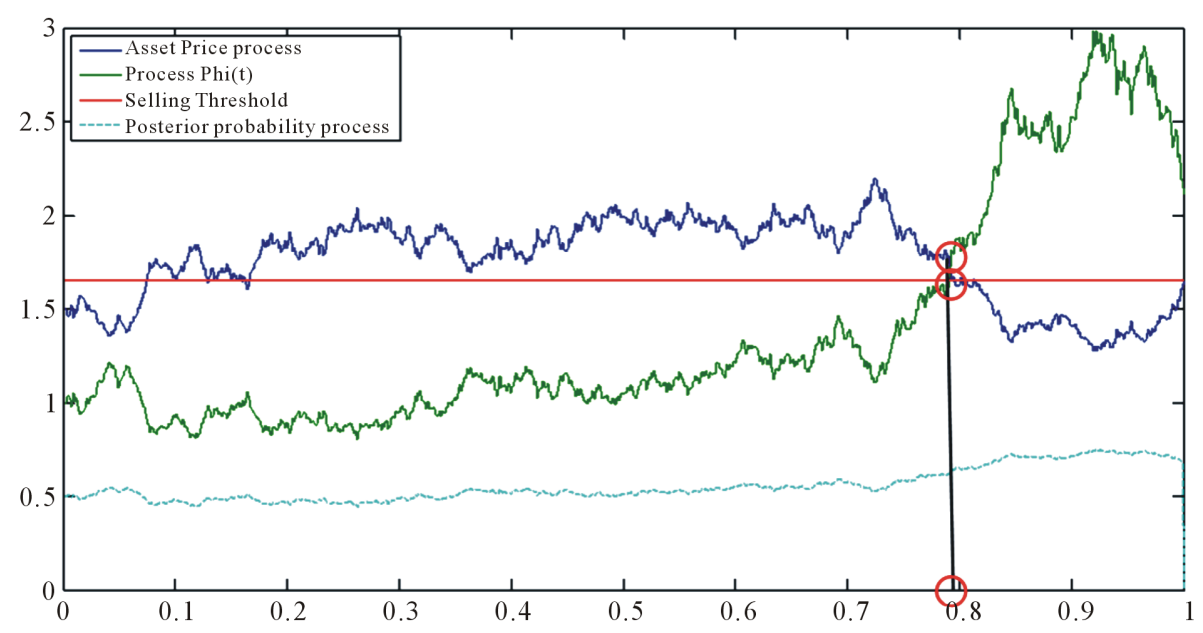

Figure 7. A simulation of asset price process, the posterior probability process, process $\Phi(t)$, the threshold probability and the optimal stopping time. In this case, the first time that the process $\Phi(t)$ over passes the threshold probability at the time 0.795 so the optimal stopping time is 0.795 . 


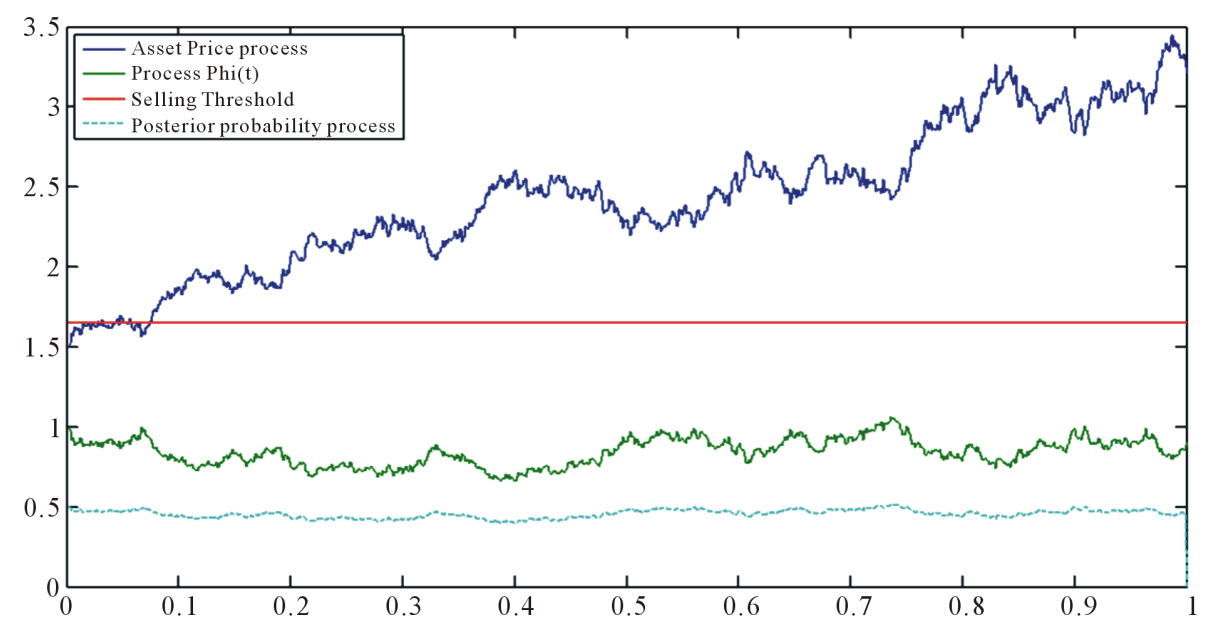

Figure 8. A simulation of asset price process, the posterior probability process, process $\Phi(t)$, the threshold probability and the optimal stopping time. In this case, the process $\Phi(t)$ always under the threshold probability so the optimal stopping time is the final time 1 , the same with the case in Figure 4.

numerical solution to the experimental selling threshold. In next studies, we will consider problems in which the price growth rate is a Markov process which has more than 2 states and establish some properties as well as distribution of stopping time.

\section{Acknowledgements}

This research is funded by Vietnam National Foundation for Science and Technology Development (NAFOSTED) under grant number 10103-2012.17.

\section{References}

[1] Khanh, P. (2014) Optimal Stopping Time to Buy an Asset When Growth Rate Is a Two-State Markov Chain. American Journal of Operations Research, 4, 132-141. http://dx.doi.org/10.4236/ajor.2014.43013

[2] Khanh, P. (2012) Optimal Stopping Time for Holding an Asset. American Journal of Operations Research, 4, 527-535. http://dx.doi.org/10.4236/ajor.2012.24062

[3] Peskir, G. and Shiryaev, A.N. (2006) Optimal Stopping and Free-Boundary Problems (Lectures in Mathematics ETH Lectures in Mathematics. ETH Zürich (Closed)). Birkhäuser, Basel.

[4] Shiryaev, A.N., Xu, Z. and Zhou, X.Y. (2008) Thou Shalt Buy and Hold. Quantitative Finance, 8, 765-776. http://dx.doi.org/10.1080/14697680802563732

[5] Guo, X. and Zhang, Q. (2005) Optimal Selling Rules in a Regime Switching Model. IEEE Transactions on Automatic Control, 9, 1450-1455. http://dx.doi.org/10.1109/TAC.2005.854657

[6] Lipster, R.S. and Shiryaev, A.N. (2001) Statistics of Random Process: I. General Theory. Springer-Verlag, Berlin, Heidelberg.

[7] Shiryaev, A.N. (1978) Optimal Stopping Rules. Springer Verlag, Berlin, Heidelberg. 